\title{
Designing pH-responsive and dielectric hydrogels from cellulose nanocrystals
}

\author{
XIAOYUAN GAO, KISHOR KUMAR SADASIVUNI, HYUN-CHAN KIM, SEUNG-KI MIN and \\ JAEHWAN KIM* \\ Center for EAPap Actuator, Dept. of Mechanical Engineering, Inha University, 253 Yonghyun-Dong, \\ $\mathrm{Nam}-\mathrm{Ku}$, Incheon 402-751, South Korea \\ e-mail: jaehwan@inha.ac.kr
}

MS received 3 December 2014; revised 24 February 2015; accepted 28 February 2015

\begin{abstract}
We report the fabrication and characterization of a pH-responsive hydrogel with improved mechanical and dielectric properties from cellulose nanocrystals. X-ray diffraction and SEM observations were used to analyze the sample morphology. The resulting $\mathrm{pH}$ detector exhibits a pronounced change in their swelling index in response to variation in $\mathrm{pH}$. It was used singly and in combination with other nanomaterials to optimize smart material designs. The applications of the developed material are anticipated in chemical, environmental and biological systems.
\end{abstract}

Keywords. Cellulose; Cellulose Nanocrystals; Hydrogels; pH-responsive; Dielectric property

\section{Introduction}

Cellulose is the most abundant and low-cost natural polymer on earth. By virtue of its non-toxicity, biocompatibility and degradability cellulose has broad applications in the food, pharmaceutical and chemical industries. However, economically feasible and environmentally friendly chemical processes are required for more efficient utilization of cellulose. ${ }^{1,2}$ These properties make cellulose a soft electroactive material. The cellulose nanocrystals (CNCs) obtained from cellulose possess low density, high mechanical strength, thermal stability, and chemical resistance. ${ }^{3}$ CNCs can be isolated at a low cost from renewable natural sources such as cotton, wood, bananas, tunicates, and many other species. ${ }^{4-7}$ They have a typical diameter of 5$50 \mathrm{~nm}$ and a length of 100-3000 nm, depending on the source and the processing conditions. The rodlike, highly crystalline CNCs are synthesized by breaking natural cellulose fibers with acid hydrolysis These CNC nanoparticles show good piezoelectric response making them applicable in sensors and actuators and biomedical fields. ${ }^{8}$

The interest in nanoparticle $(<100 \mathrm{~nm})$ filled polymer composites has emerged tremendously in recent years. ${ }^{9}$ The CNCs that are derived from abundant and renewable sources have drawn increasing attention in this regard due to their high aspect ratio,

*For correspondence low density, and eco-friendliness. ${ }^{10-12}$ Many reports have come out in which the CNCs enhance the polymer properties. ${ }^{13}$ For instance the Young's modulus of extruded starch plastic was increased significantly when $10.3 \%(\mathrm{w} / \mathrm{w})$ CNC derived from cotton was added. ${ }^{14}$ A 5\% CNC loading of the solvent casted polyurethane film increased its tensile strength and strain from 39 to $257 \mathrm{MPa}$ and 157 to $237 \%$, respectively. ${ }^{15}$ The amphiphilic CNCs can be easily functionalized as well for achieving better compatibility with the polymer matrix. As mechanical and dielectric properties of the nanocomposite fibers are governed by the interfaces between the nanofillers and polymer matrix, stronger interactions are more beneficial. ${ }^{16}$ By incorporating CNCs into polyacrylamide, abundant hydrogen bonding between the hydroxyl groups on the CNC surfaces and carboxylic acids along the polymer chain facilitated the dispersion of CNCs in the polymer solution.

The surface modifications of $\mathrm{CNCs}$ for $\mathrm{pH}$-sensing have also been investigated ${ }^{17}$ and several of these have been extensively researched in terms of miniaturized pH sensor. ${ }^{18-20}$ The $\mathrm{pH}$ value is a quantity commonly used to monitor the properties of solutions, and it is one of the most critical measurable parameters in many fields such as medicine, chemistry, biology and material science. ${ }^{21}$ A popular approach that exploits metal oxide as $\mathrm{pH}$ sensor is the ion sensitive field effect transistor. ${ }^{22}$ In contrast to metal oxide and other toxic carbon materials, whose acute toxicity precludes their utilization in vivo, CNCs appear to be rather benign. ${ }^{23-25} \mathrm{In}$ 
addition, strong hydrophilicity of cellulose promises reduced nonselective adsorption of biological materials that often causes degradation of transducer surfaces. ${ }^{26}$ The advantages of biodegradable cellulose/CNC hydrogels include its applicability in controlling the size with $\mathrm{pH}$ variation and the electric responsive character simultaneously due to the electromechanical nature of cellulose.

Here, we introduce a pH-responsive and highly dielectric hydrogel where cellulose forms the base matrix and CNCs the filler. The morphology and structural property relationship of the cellulose/CNC hydrogels were determined with the help of X-ray diffraction (XRD) studies and scanning electron microscopy (SEM). The proposed cellulose/CNC hydrogel works as a strong transparent $\mathrm{pH}$-responsive hydrogel and a good dielectric material.

\section{Experimental}

\subsection{Materials}

Cotton pulp (MVE, DPw-4580) was supplied by Buckeye Technologies Co. Ltd. (US). N,N-Dimethylacetamide (DMAc), sulphuric acid $\left(\mathrm{H}_{2} \mathrm{SO}_{4}\right)$, and sodium hydroxide $(\mathrm{NaOH})$ were procured from Sigma-Aldrich, South Korea. Lithium chloride $(\mathrm{LiCl})$ and Isopropanol (IPA) were purchased from Dae Jung chemicals \& metals Co. Ltd.

\subsection{Preparation of cellulose solution}

The preparation of cellulose solution using DMAc/LiCl solvent has been reported previously and this is a brief summary. ${ }^{27}$ The cellulose (1.5\%) was dissolved in the mixed solvent system of $\mathrm{DMAc} / \mathrm{LiCl}$ with $\mathrm{LiCl}$ concentration $8.5 \%$. Then the mixed pulp with $\mathrm{DMAc} / \mathrm{LiCl}$ was heated at $80^{\circ} \mathrm{C}$ for 3 hours with mechanical stirring. After that, by raising the reaction temperature to $150^{\circ} \mathrm{C}$ and keeping the temperature for $15 \mathrm{~min}$ the heater was turned off and allowed slowly to reach room temperature with stirring for 24 hours. Then the reaction temperature was raised to $100^{\circ} \mathrm{C}$, kept it for $6 \mathrm{~h}$, then turned off the heater and let it slowly cool to room temperature. Thereafter, stirred for $12 \mathrm{~h}$ and kept it in a bottle leaving for at least 3 weeks for aging. Finally, the pure and transparent cellulose solution was obtained through centrifuging process.

\subsection{Synthesis of $\mathrm{CNC}$}

The CNC nanoparticles used in this study were prepared by $\mathrm{H}_{2} \mathrm{SO}_{4}(175 \mathrm{~mL}$ of $30 \%$ (v/v) with water) for hydrolysis of $98 \%$ pure cotton pulp $(20.0 \mathrm{~g})$ under mechanical stirring $(200 \mathrm{rpm}, 6 \mathrm{~h})$ at $60^{\circ} \mathrm{C}$. An alkaline $(1 \mathrm{M} \mathrm{NaOH})$ treatment on the cotton cellulose fiber was also carried out to remove the non-cellulosic components prior to acid hydrolysis. The hydrolysis left a suspension which was further successively centrifuged with distilled water to attain neutrality and then homogenized (10 min, $11000 \mathrm{rpm})$ and dialyzed overnight.

\subsection{Fabrication of cellulose/CNC hydrogels}

Initially, CNC in 3 and $5 \mathrm{wt} \%$ dispersed in DMAc by 3 hours of sonication was mixed with cellulose/DMAc solution and cast into a petri dish, followed by curing with the mixture of deionized water and IPA in a ratio of 4:6 for $3 \mathrm{~h}$. This slow curing process prevents aggregation of $\mathrm{CNCs}$ and eliminates remnant $\mathrm{LiCl}$ and DMAc. Finally the cured hydrogel was rinsed in two different deionized water baths for making hydrogel of $\mathrm{pH}=7$. For SEM, XRD, mechanical pull test, UV-visible and dielectric constant measurements, the solution was cast on glass using doctor blade and dried in a vacuum oven overnight at $60^{\circ} \mathrm{C}$, resulting in film formation.

\subsection{Characterization}

Sample morphology was checked with the help of AFM, TEM and SEM. The FE-TEM micrographs of the CNCs were analyzed with JEOL, JEM 2100F and the FE-SEM images of the hydrogel films with a field emission scanning electron microscope (Hitachi S-4300 microscope). XRD patterns were checked with a thin film X-ray diffractometer using $\mathrm{CuK} \alpha$ target radiation at $40 \mathrm{kV}$ and $50 \mathrm{~mA}$, at a scanning rate of $0.015 \% \mathrm{~min}$. The diffraction angle was varied from 5 to $40^{\circ}$. Optical transmittance of the samples was studied using UV-visible spectrophotometer. For this the spectra of the films in the range of $200-800 \mathrm{~nm}$ were recorded with a Hewlett Packard (8452A) diode array. The mechanical test was conducted according to ASTM D-882-97 using a universal testing machine. The samples were put between two plates; the upper plate would press the sample until it reached a fixed value. Test was performed under room temperature and at compressing rate of $0.005 \mathrm{~mm} / \mathrm{s}$. Dielectric constant was measured using HP 4284A LCR meter between 20 and $4000 \mathrm{~Hz}$ frequency with $1 \mathrm{~V}$. The hydrogels were cast as films using a doctor blade and gold electrodes were coated on both sides of them. 

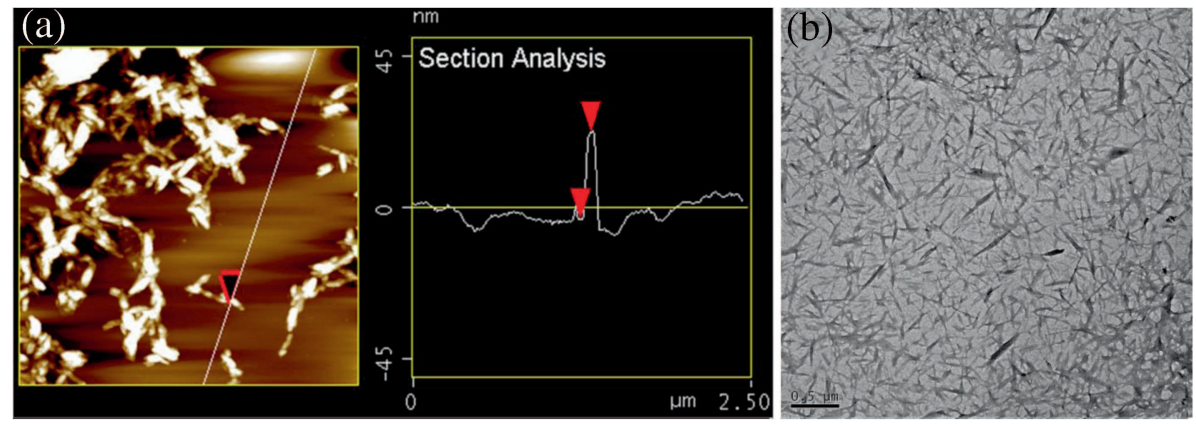

Figure 1. (a) AFM and (b) TEM images of the synthesized CNC.

\section{Results and Discussion}

\subsection{Sample Morphology}

As given in the AFM of synthesized CNCs in figure 1a, they exhibit rod like nanostructures with 25$40 \mathrm{~nm}$ diameter. For taking AFM, CNCs were spin coated on the silicon wafer which is oxidized by piranha solution. The compact network structure of CNCs reveals the internal bondings present in it. Figure $1 \mathrm{~b}$ illustrates the high resolution TEM (FE-TEM, JEOL, JEM 2100F) micrograph of a very dilute suspension of CNC (in Isopropanol $0.01 \% \mathrm{w} / \mathrm{v}$ ) deposited on carboncoated grids. The diameter of the 'rod-like' nanocrystals has wide range of distribution but the length of most of the nanocrystals lies within the range $250-380 \mathrm{~nm}$.

The morphology of hydrogel was investigated by the SEM images of the pristine cellulose and its hydrogels with CNCs. Figure 2 illustrates the SEM images of the pristine cellulose and cellulose/CNC hydrogels with 3 and $5 \mathrm{wt} \%$ of incorporated CNCs. The hydrogels with $\mathrm{CNC}$ loadings ( 3 and $5 \mathrm{wt} \%$ ) exhibit uniform dispersion of CNCs within the cellulose matrix, where CNCs are closely attached on the polymer matrix surface thus reinforcing the network.

One can note that the neat cellulose exhibited a relatively rough structure (figure $2 \mathrm{a}$ ). On the other hand cellulose/CNC hydrogel surfaces are smooth, which is attributed to the attachment of CNCs on cellulose and its smoothness increased with an increase of the filler loading (figures $2 \mathrm{~b}$ and $2 \mathrm{c}$ ). In the cellulose/CNC hydrogel, the cellulose chains are physically linked together with strong interaction between the CNCs leading to progressively larger branches. This physical hydrogel obtained by an exceptionally simple method of mixing cellulose and $\mathrm{CNC}$ before network formation is schematically represented in figure 3 and the interactions between cellulose chains are effectively permanent at a given set of experimental conditions. Energetically favorable interactions existed between adjacent cellulose-CNCs, by which the interconnected network structure with a repeated distance of a few hundred nanometers was formed. Since the CNCs possess hydrophilic character they are inherently compatible with $\mathrm{DMAc} / \mathrm{LiCl}$ cellulose system and this makes the homogeneous dispersal of surface charged CNCs in DMAc relatively easy. This is clear from the SEM images of figure 2 where the CNCs are uniformly dispersed within the cross-linked network under the swollen state. Formation of the cross-linked network with a small amount of CNCs indicates that the CNCs act as multifunctional cross-links with highly effective functionality.

Figure 4 shows the XRD patterns of the pristine cellulose, cellulose/CNCs with 3 and 5 wt $\%$ and pure CNCs. CNCs display four well-defined diffraction peaks at $14.7,16.5,22.7$, and 34.5 , which are typical of cellulose I. ${ }^{8}$ Compared with those of CNCs, the mainly
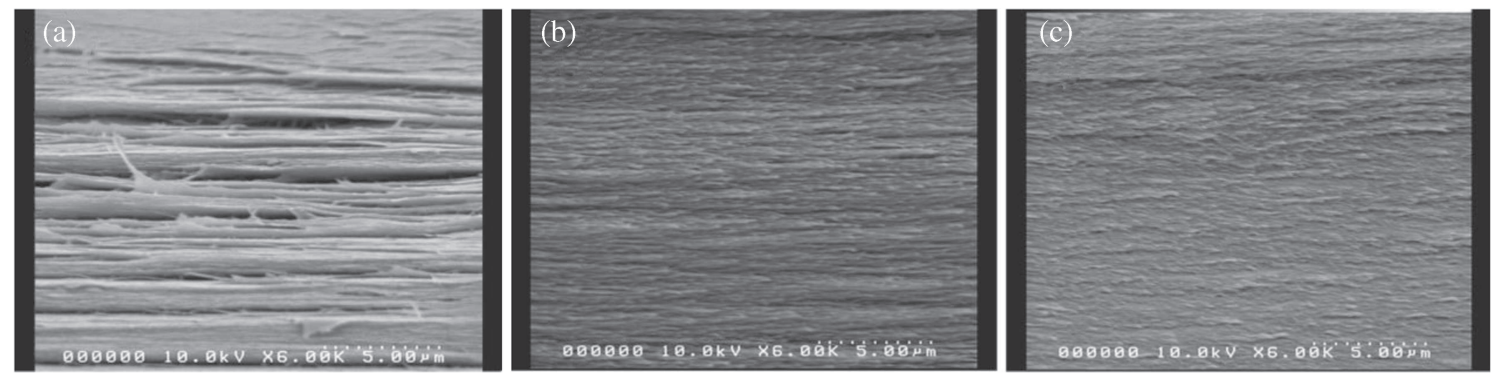

Figure 2. SEM analysis of (a) cellulose, (b) cellulose/CNC with $3 \%$ loading and (c) $5 \%$ loading. 


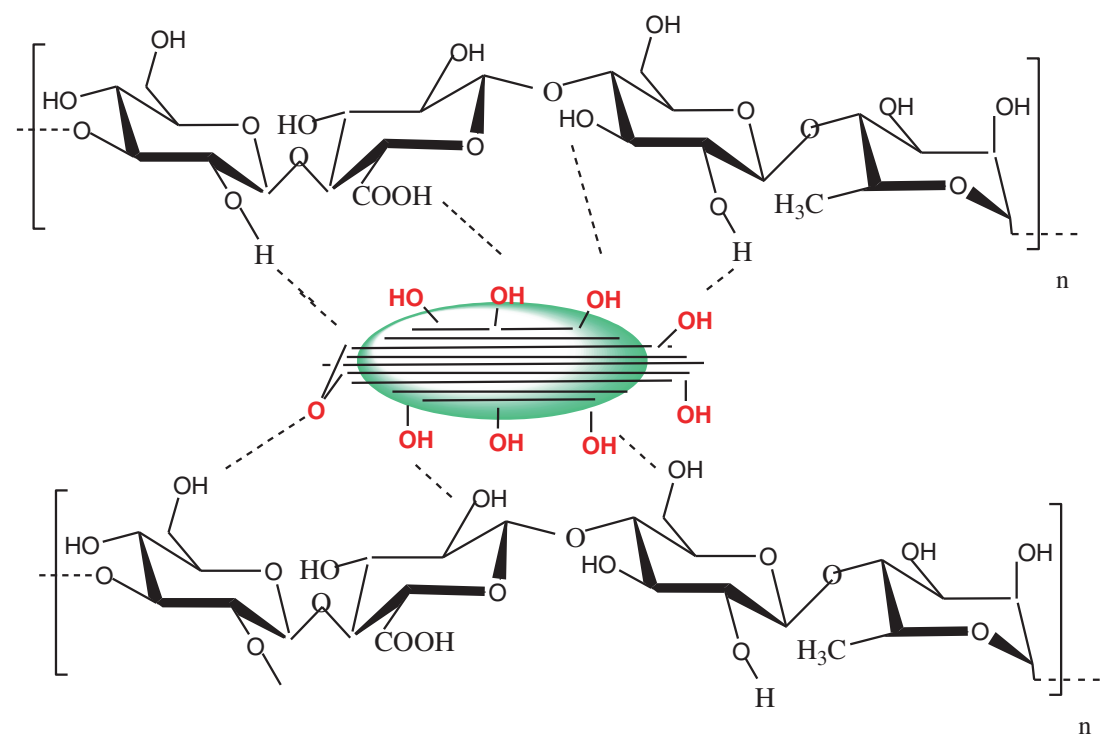

Figure 3. Interaction between $\mathrm{CNC}$ and cellulose.

strong crystalline peak appeared at around $2 \theta=21.8^{\circ}$ for the pristine cellulose and cellulose/CNCs However, in the case of c cellulose/CNCs there are no characteristic peaks of CNCs. Moreover the introduction of CNCs has a negligible effect on the crystallinity of the materials based on the full width at half maxima values (FWHM).

\subsection{UV-Transmittance of the cellulose and cellulose/CNCs hydrogel films}

Figure 5 represents the UV-vis spectra and the appearance of the pristine cellulose and cellulose/CNC hydrogel films. The hydrogel film must be transparent as an optical film as they showed 88-85\% (at $550 \mathrm{~nm}$ ) transmittance. The given transmittance versus wavelength

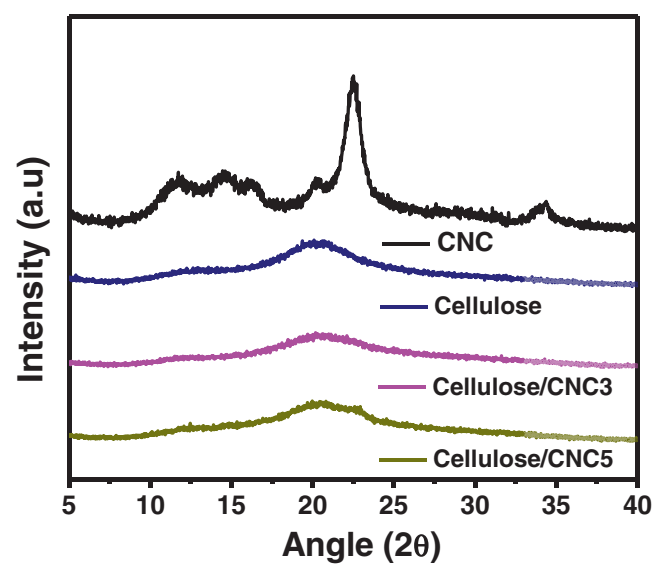

Figure 4. XRD analysis of CNC, cellulose and cellulose/CNC. plots shows an increase in transmittance at 200-800 $\mathrm{nm}$, which is approximately the lower limit of the visible region. These observations suggest an interface free structure for the materials. In other words, CNC has strong reinforcing effect and it is well dispersed in the cellulose matrix. The very small size of the nanocrystals and the low filler content (as evidenced from the AFM and TEM in figure 1) make light scattering through the film easy.

\section{$3.3 p^{H}$ response of cellulose and cellulose/CNC hydrogel}

The swelling indices of the cellulose and cellulose/CNC $\mathrm{pH}$ sensors calculated using Equation 1 are shown in figure 6. When CNCs were dispersed in the hydrogel,

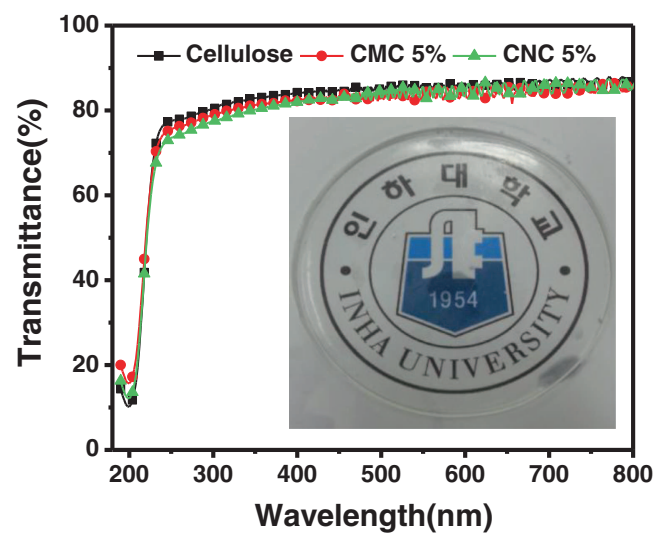

Figure 5. Optical transparency of cellulose and its cellulose/CNC hydrogels (Inset Optical image of cellulose/CNC5 hydrogel). 


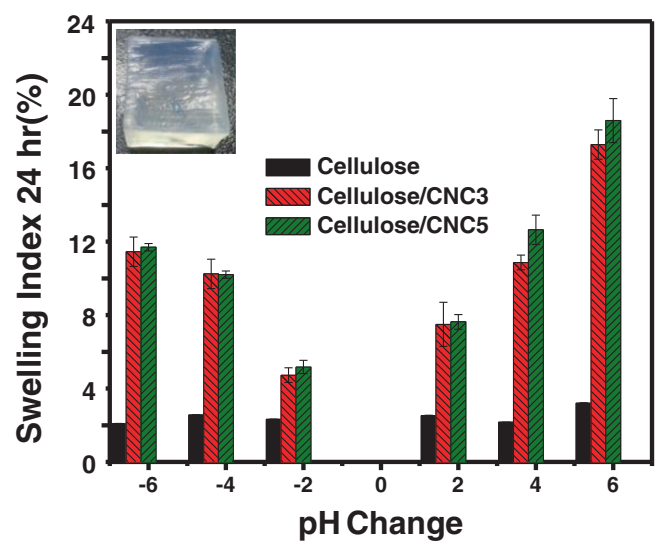

Figure 6. $\mathrm{P}^{\mathrm{H}}$ sensing of cellulose and cellulose/CNC hydrogels at different $\mathrm{pH}$ (Initial $\mathrm{pH}$ of samples is 7).

we observed significant changes in swelling index as the $\mathrm{pH}$ of the solution varied:

$$
\text { SwellingIndex }=\frac{W_{2}-W_{1}}{W_{1}} \times 100
$$

where $W_{2}$ and $W_{1}$ are the weights of the specimen before and after swelling. $W_{2}$ is weight of the sample at $\mathrm{pH} 7$ and $W_{1}$ the weight of the sample at a different $\mathrm{pH}$.

Results of structural characterization indicate that the incorporated $\mathrm{CNCs}$ interact strongly with the cellulose matrix through hydrogen bonding. The $\mathrm{pH}$ sensitivity of the cellulose and cellulose/CNC can be divided into two linear regions depending on the acidic $\mathrm{pH}$ level. The hydrogels show a linear response with low sensitivity between $\mathrm{pH} 1$ to 6 . However, when the $\mathrm{pH}$-responsive hydrogel senses alkaline buffer solution between $\mathrm{pH} 7$ to 12 , slope of the linear curve increases. Nevertheless, the hydrogel response to $\mathrm{pH}$ value is changed significantly compared to cellulose. For the $\mathrm{pH}$ sensitive hydrogels reported in this paper, functional groups are bound to the chains. Hydrogels are capable of undergoing large reversible deformations in response to changes in several environmental factors. ${ }^{28}$ We maintained the temperature constant during measurements. Special modification of the polymer structure can also lead to gel formation that is $\mathrm{pH}$ sensitive to specific biological agents. ${ }^{29}$ Swelling/deswellingphenomena occur for a hydrogel containing functional groups bound to its polymer chains when the $\mathrm{H}^{+}$or $\mathrm{OH}^{-}$ comes off in basic solutions and combines with counter ion. The charge is compensated by ions that enter into the gel together with counter ions and the charge maintains neutrality. The increased ionic concentration gives rise to an osmotic pressure that causes the gel to swell/deswell. For a better understanding we considered acidic medium, in which the conjugate base of the buffer reversibly binds hydrogen ions in regions

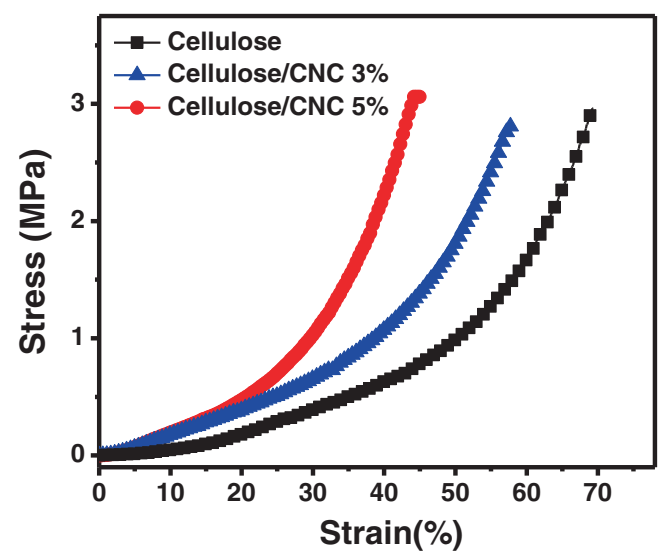

Figure 7. Compressive stress-strain curves of different hydrogels.

of higher concentration and releases the hydrogen ion after diffusing to a region of lower concentration. ${ }^{30}$ Equilibrium of hydrogel occurs when the elastic restoring force of the network balances the osmotic forces and swelling index becomes constant. ${ }^{30-32}$

\subsection{Mechanical Properties}

The mechanical properties of cellulose hydrogels with two different concentrations (CNC 3\% and 5\%) were investigated. Figure 7 illustrates the stress-strain curves. It is found that the addition of $\mathrm{CNC}$ in the cellulose hydrogel improves the mechanical property of the pristine cellulose hydrogel. Compared with the pristine cellulose hydrogel, the cellulose/CNC5 hydrogel exhibited significant improvement in Young's modulus: the Young's modulus increased from $0.40,0.48$ to $0.55 \mathrm{GPa}$ for cellulose, cellulose/CNC3 and cellulose/CNC5. The high modulus is related to the high cross-linking density, where the multifunctional physical cross-links of $\mathrm{CNCs}$ form at the interface with cellulose matrix as per the schematic representation in figure 3. Owing to the strong interaction of CNCs through surface hydroxyl groups, they have pronounced tendency for self-association, which is advantageous for the formation of load-bearing percolating networks within the host polymer matrix. ${ }^{8}$ It is considered that the interactions between polymer-polymer, polymer-filler, and filler-filler contribute to the mechanical properties of the hydrogels.

\subsection{Dielectric properties}

In order to check the dielectric response of the samples, dielectric constant, $\varepsilon^{\prime}$ of the cellulose hydrogels along with the $\mathrm{CNC}$ inclusion was measured at $25^{\circ} \mathrm{C}$ 


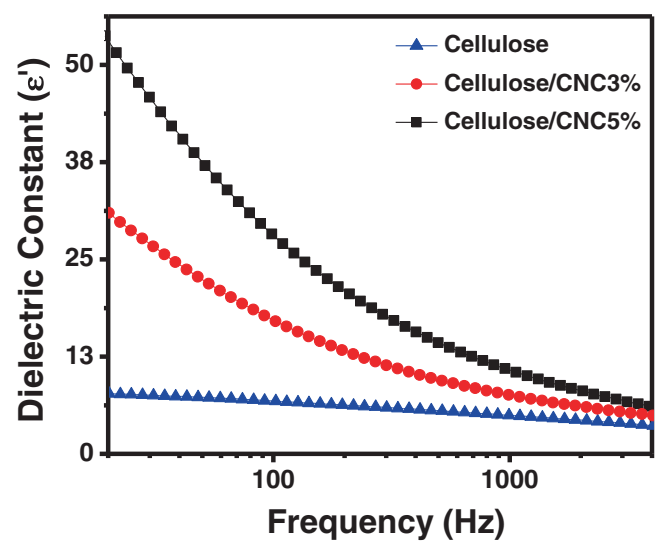

Figure 8. Dielectric property of cellulose and cellulose/CNC hydrogels.

temperature, $25 \%$ relative humidity. Figure 8 shows the measurement results The dielectric measurements show that the incorporation of the CNCs in the cellulose matrix increases $\varepsilon^{\prime}$. When the values are compared, CNC possesses enhanced dielectric constant which is attributed to the motion of free charge carriers due to interfacial polarization effects and charges present around the CNC surface. This is possible due to the presence of moderate number of oxide functionalities (charge centers) and conjugation (carrier centers) on the CNC surface. The $\varepsilon^{\prime}$ of a microelectronic dielectric polymer depends on the amount of mobile (polarizable) electrical charges in it and the degree of mobility of these charges. ${ }^{33-36}$ According to MaxwellWagner-Sillars (MWS) process polymer-filler interfacial interaction, like donor-acceptor complexes is necessary to get changes in the composite dielectric properties. The huge interfacial area of CNCs in the cellulose matrix provides numerous sites for the reinforced MWS effect. $^{37-39}$

\section{Conclusions}

In summary, the cellulose/CNC hydrogels were prepared by solvent blending method and a strong and transparent $\mathrm{pH}$-responsive hydrogel with good dielectric behavior has come out from this study. The results of SEM and WAXD techniques indicate homogeneous nanostructures for the obtained hydrogels and a strong hydrogen bonding interaction between CNCs and the cellulose matrix. Though long term stability in harsh environment and industrial detection protocols to provide complementary sensing information are the issues in sensor technology, the cellulose based devices have great potential for cheap, disposable and biocompatible sensors. The maximum transmittance reaches $88.7 \%$ at $800 \mathrm{~nm}$ for the $\mathrm{CNC} /$ cellulose hydrogels similar to the pristine cellulose hydrogel. CNC/cellulose hydrogels also illustrate significant improvement in the dielectric constant and the reported $\mathrm{pH}$ responsive hydrogels are thus useful in energy storage applications as well.

\section{Acknowledgements}

This work was supported by the National Research Foundation of Korea (NRF- 2013M3C1A3059586).

\section{References}

1. Yin C Y, Li J B, Xu Q, Peng Q, Liu Y B and Shen X Y 2007 Carbohydr. Polym. 67147

2. Kim J Yun S and Ounaies Z 2006 Macromolecules 39 4202

3. Mariano M, El Kissi N and Dufresne A 2014 J. Polym. Sci., Part B: Polym. Phys. 52791

4. Kumar S, Hofmann M, Steinmann B, Foster E J and Weder C 2012 ACS Appl. Mater. Interfaces 45399

5. Azizi Samir M A, Alloin F and Dufresne A 2005 Biomacromolecules 6612

6. Mueller S, Weder C and Foster E J 2013 RSC Adv. 10 907

7. Marchessault R H, Morehead F F and Walter N M 1959 Nature 184632

8. Sadasivuni K K, Kafy A, Zhai L, Ko H U, Mun S and Kim J 2014 Small 101002

9. Siqueira G., Bras J and Dufresne A 2009 Biomacromolecules 10425

10. Favier V, Chanzy H and Cavaille J Y 1995 Macromolecules 286365

11. Samir M A S A, Alloin F, Sanchez J Y and Dufresne A 2004 Macromolecules 1537

12. Samir M A S A, Alloin F and Dufresne A 2005 Biomacromolecules 6612

13. Eichhorn S J, Dufresne A, Aranguren M, Marcovich N E, Capadona J R, Rowan S J, Weder C, Thielemans W, Roman M, Renneckar S, Gindl W, Veigel S, Keckes J, Yano H, Abe K, Nogi M, Nakagaito A N, Mangalam A, Simonsen J, Benight A S, Bismarck A, Berglund L A and Peijs T 2010 J. Mater. Sci. 451

14. Orts W J, Shey J, Imam S H, Glenn G M, Guttman M E and Revol J F 2005 J. Polym. Environ. 13301

15. Henriksson M U Q, Liu X and Berglund L A 2007 Biomacromolecules 83687

16. Renneckar S, Zink-Sharp A, Esker A R, Johnson R K and Glasser W G 2006 ACS Symp. Ser. 93878

17. Nielsen L J, Eyley S, Thielemans W and Aylott J W 2010 Chem. Commun. 474177

18. Samuel J, Strinkovski A, Shalom S, Lieberman K, Ottolenghi M, Acnir D and Lewis A 1994 Mater. Lett. 21431

19. Roy S G, Haldar U and De P 2014 ACS Appl. Mater. Interfaces 64233

20. Patil N, Soni J, Ghosh N and De P 2012 J. Phys. Chem. B 11613913

21. Seari L A and Seitz W R 1982 Anal. Chem. 54821

22. Fulati A, Usanman Ali S M, Riaz M, Amin G, Nur O and Roth M 2006 J. Electroanal. Chem. 58672 
23. Lam C W, James J T, McCluskey R, Arepalli S and Hunter R L 2006 Crit. Rev. Toxicol. 36189

24. Clift M J D, Foster E J, Vanhecke D, Studer D, Wick P, Gehr P, Rothen-Rutishauser B and Weder C 2011 Biomacromolecules 123666

25. Dong S, Hirani A A, Colacino K R, Lee Y W and Roman M 2012 Nano Life 211

26. Edwards J V, Prevost N, French A, Concha M, DeLucca A and Wu Q 2013 Engineering 520

27. Sadasivuni K K, Yadav M, Gao X, Mun S and Kim J 2014 Proc. SPIE 9060 Nanosensors, Biosensors, and Info-Tech Sensors and Systems 101117

28. Shukla N B and Madras G 2011 Ind. Eng. Chem. Res. 5010918

29. Roy S G and De P 2014 Polymer 555425

30. Chu Y, Varanasi P P, McGlade M J and Varanasi S 1995 J. Appl. Polymer Sci. 582161

31. Grimshaw P E, Grodzinsky A J, Yarmush M L and Yarmush D M 1990 Chem. Eng. Sci. 452917
32. Ruckenstein E and Sasidhar V 1984 Chem. Eng. Sci. 39 1185

33. Sadasivuni K K, Ponnamma D, Kumar B, Strankowskie M, Cardinaels R, Moldenaers P, Thomas S and Grohens Y 2014 Compos. Sci. Technol. 10418

34. Sadasivuni K K, Castro M, Saiter A, Delbreilh L, Feller J F, Thomas S and Grohens Y 2013 Mater. Lett. 96109

35. Kafy A, Sadasivuni K K, Kim H-C, Akther A and Kim J 2014 Phys. Chem. Chem. Phys., DOI: 10.1039/C4CP05921B

36. Sadasivuni K K, Saiter A, Gautier N, Thomas S and Grohens Y 2013 Colloid Polym. Sci. 2911729

37. Sadasivuni K K, Ponnamma D, Thomas Sabu and Grohens Y 2014 Prog. Polym. Sci. 39749

38. Ponnamma D, Sadasivuni K K, Grohens Y, Guo Q and Thomas S 2014 J. Mater. Chem. C 28446

39. Yuan J, Yao S, Dong Z, Sylvestre A, Genestoux M and Bai J 2011 J. Phys. Chem. 1155515 